\title{
Un dernier édito
}

Entré au Comité de rédaction de notre revue, Radioprotection, en 1991 (Vol. 26 (3)) j’ai signé mon premier éditorial en 1996 (Vol. 31 (4)), lorsque j'ai succédé au regretté Daniel BLANC, au poste de Président du Comité de rédaction. Ce numéro fut pour moi aussi délicat qu'exceptionnel et émouvant, dans la mesure où il convenait de consacrer un hommage légitime à Henri JAMMET disparu peu avant.

Aujourd'hui, 18 ans après, je passe le flambeau reçu de Daniel BLANC à François PAQUET, qui je l'espère, n'aura pas, à inaugurer sa fonction par une rubrique nécrologique! On peut mieux faire... Je quitte ce poste serein et confiant; François a démontré depuis bien longtemps, et dans de fort nombreux domaines, ses qualités scientifiques et humaines alliées à une rigueur et une exigence intellectuelle qui en feront un excellent Président. J'aimerais d'ailleurs rappeler qu'il m'a également succédé au Comité 2 de la CIPR. Ces passations deviennent une habitude, me direz-vous. J'acquiesce volontiers, certain qu'il s'agit là d'une bonne habitude.

De 1996 à 2014, il s'est passé beaucoup de choses, et vous l'avez constaté, la revue a beaucoup changé. Tout ce travail a pu se faire grâce au Comité de rédaction, à notre éditeur EDP-Sciences, au Secrétaire général de la SFRP, Jacques LOMBARD puis à notre Directrice de cette même société, Valérie CHAMBRETTE (seulement deux ans avec Valérie, mais deux années très riches). Bien entendu, sans le soutien du Conseil d'Administration et ses différents Présidents rien n'aurait été vraiment possible. À ce titre, je tiens à remercier tout particulièrement Laurent STRICKER qui m'avait demandé d'exposer ma vision de la gestion de cette revue et qui, au vu de mes réponses, m'a accordé sa confiance en me demandant de succéder à Daniel BLANC. Il souhaitait ardemment que toutes les composantes de la radioprotection française soient représentées au sein de ce Comité, et cette pluralité ne cesse d'enrichir notre exigence grâce aux regards croisés ainsi posés sur les articles soumis.

En ce moment de remerciements, il convient de saluer le travail constructif accompli avec EDP-Sciences, notre fidèle éditeur, et de remercier tout particulièrement Agnès HENRI, un soutien indéfectible et une conseillère avisée. L'idée d'une gestion en ligne, c'est elle, l'idée d'une nouvelle maquette, c'est elle encore. Que de talent, d'efficacité et de professionnalisme ! N'oublions pas toutes ses collègues, elles se reconnaîtront.

Au sein du Comité de rédaction, j'ai également particulièrement apprécié le soutien indéfectible et l'aide de notre viceprésidente, Jeannine LALLEMAND, qui a pu faire croire à certains que j'écrivais dans un langage parfait; non ce n'était pas seulement moi. Son rôle ne s'est pas arrêté là, rassurez-vous.

Il faut également remercier les auteurs, c'est une évidence, mais aussi les relecteurs venant du monde entier, véritables garants de l'exigence et de la rigueur qu'une revue avec un Comité de lecture requiert.

Après le miel, un peu de vinaigre. Quels furent mes déceptions ou mes agacements? Je ne les citerai pas tous mais prendrai un seul exemple, à savoir l'échec de mes nombreuses sollicitations auprès des orateurs des journées organisées par la SFRP. Que d'excellents articles manqués suite à des brillantes présentations, innovantes ou relatives au quotidien de la radioprotection. Ces articles auraient pourtant pu être répertoriés dans les bases internationales et donc accessibles aux experts internationaux. Malheureusement, il n'en fut rien ou presque. Je souhaite que, sur ce point François, avec l'aide de Valérie, réussisse mieux que moi, je n'en doute pas. Vous voyez qu'il lui reste beaucoup de travail pour améliorer encore notre revue, et je reste optimiste.

Merci également à tous ceux que j'ai côtoyés durant ces 18 ans de présidence et que le format contraint de cet éditorial ne me permet pas de citer. Si, deux : Janine CERVERA et Patricia PIQUERAS sans qui gérer la SFRP serait bien difficile.

Cette revue restera toujours dans mon cœur. Bon vent à Radioprotection et à la SFRP et pour ne pas déroger à une vieille et bonne tradition: À vos plumes.

Enfin, avant de clore ce dernier édito, il me faut, tradition oblige, souhaiter à tous nos lecteurs une excellente année 2015. 